\title{
Evaluation of Potential Residual Silica as Antireflective Layer for Selective Solar Surface of Black Chromium
}

\author{
Ithyara Dheylle Machado Medeiros ${ }^{a}$, Ronny Peterson da Nobrega Gonçalves ${ }^{a}{ }^{\circledR}$, Valeska Lisandra \\ Menezes $^{a}$, Gabriela Oliveira Galvão ${ }^{b}$, Kelly Cristiane Gomes ${ }^{a, b} * \mathbb{D}$ \\ aPrograma de Pós-Graduação em Engenharia Mecânica, Universidade Federal da Paraíba - UFPB, \\ João Pessoa, PB, Brasil \\ ${ }^{b}$ Departamento de Engenharia de Energia Renovável, Universidade Federal da Paraíba - UFPB, João \\ Pessoa, PB, Brasil
}

Received: November 22, 2018; Revised: March 15, 2019; Accepted: April 08, 2019

\begin{abstract}
Selective surfaces have the function of absorbing strongly the sunlight, while ideally losing little heat to the environment via convection and radiation. The present study sought to obtain a selective surface of black chromium coated with an antireflective (AR) layer based on sugarcane bagasse ash using copper as a substrate. From this selective surface the electrodeposition parameters (time, working distance, voltage) as well as the surface behavior were analyzed in order to determine if the AR layer provided an increase in the absorption levels of the film. By means of the present study it was possible to conclude that, in terms of the electrodeposition parameters evaluated, the anode-cathode distance of $5 \mathrm{~cm}$ stimulated the obtaining of higher levels of absorption. The addition of the antireflective layer on the black chromium films favored the increase of the absortance average in all the adopted parameter sets, besides reducing the standard deviation around the average. As for the microstructure of the obtained films, the increase of the parameter $R a$ promoted the increase of the absorption, due to the formation of optical traps.
\end{abstract}

Keywords: residual silica, antireflective layer, selective surfaces, black chromium, solar energy.

\section{Introduction}

The energy potential of the sun is source of study of many applications ${ }^{1}$, however a methodology for a better use of this source still needs to be explored. In addition, the efficiency of the collection, conversion and storage for the most possible economic exploitation of this energy is fundamental for its utilization in commercial scale ${ }^{2}$.

An economic way of using solar energy requires the utilization of flat plane solar collectors, which capture the largest portion of the sun's incident energy and transfer a fraction of that captured energy to a working fluid that will transport it, so that it is used ${ }^{3}$.

In thermal solar technologies, the sunlight is absorbed as heat in the absorber of the flat surface. This coating must strongly absorb the sunlight, while ideally losing as little heat as possible to the environment via convection and radiation. A film that has a high level of absorption and a low emittance in the infrared (IR) region is called a spectrally selective absorber, or selective surface ${ }^{4}$. This surface has its efficiency increased when integrated with an antireflective layer (AR) and a reflective layer base (a substrate that intrinsically reflects the IR radiation $)^{5}$.

Therefore, a solar selective surface is the coating characterized by the following proprieties: high absorptance in the solar radiation spectrum range of $0,3 \mu \mathrm{m}<\lambda<2,0 \mu \mathrm{m}$

*e-mail: gomes@cear.ufpb.br and low emittance in the thermal irradiation range of $2,0 \mu \mathrm{m}<\lambda<50 \mu \mathrm{m}$ above the operating temperature of the absorber plate 6 .

Many methods had been used to prepare selective absorbers, such as electrodeposition, sputtering, evaporation, pyrolysis, painting and sol-gel ${ }^{7}$. This study opted for the use of the electrodeposition technique due to the simplicity of the equipment used and its low cost, the good optical proprieties (absorptance and emittance) of the obtained coatings, in addition to the technique being able to the realized at ambient temperature and atmospheric pressure, which makes it particularly attractive for industrial applications ${ }^{8}$.

In terms of the material to be electrodeposited, the use of black chromium as a solar selective coating received considerable attention since McDonald ${ }^{9}$ introduced and demonstrated its efficiency, being the technology of the coating production substantially increased since its first utilization.

The influence of the electrodeposition parameters on the absorptance of black chromium has been analysed ${ }^{10,11}$, such as the structure of the selective surface ${ }^{12}$. As an example, the multilayer structure, known by Lampert ${ }^{13}$ as interference stacks, stands out as a dielectric-metallic combination that acts as a selective filter for energy absorption. The desired effect of an interference stack is making particular wavelength of solar energy to be absorbed by the multiple reflection in the layers, while other wavelength, not corresponding the absorption frequency of these layers, are reflected ${ }^{3}$. 
A material that has been exploited in all types of antireflective coatings with particular emphasis on selective surfaces is silicon. In addition to having its AR function extensively studied, silica-based films are chemically inert, durable and resistant to abrasion ${ }^{14}$.

On this regard, in order to use a silica source, a material that stands out due to the high content of silicon oxide in its composition is the sugarcane bagasse ash with contents around $80 \%$, taking into account variations due to the condition of burning and milling of the $\operatorname{ash}^{15}$. This waste of the sugar and alcohol industry shows itself as a potential alternative as raw material for AR coatings.

As a result, this study sought to obtain a selective surface of black chromium using copper as a substrate and coated with an antireflective layer based on the sugarcane bagasse ash. From this selective surface the electrodeposition parameters as well as the surface behavior were analyzed in order to determine if the RA layer provided an increase in the absorption levels of the film.

\section{Experimental Techniques}

For the accomplishment of this study, copper substrates with dimensions of $20 \mathrm{~mm} \times 30 \mathrm{~mm}$ were used. The substrates were submitted to a degreasing bath, and then immersed in a VETEC diisopropyl ether $\left(\mathrm{C}_{6} \mathrm{H}_{14} \mathrm{O}\right)$ where they remained for 30 minutes, being sequentially washed in distilled water, dried and stored.

The composition of the electrolytic bath was based in the works of Daryabegy and Madmoodpoor ${ }^{16}$ and is described in Table 1. The set of variables applied in the electrodeposition and their respective nomenclatures are exposed in Table 2.

The distances specified in Table 2 refer to the separation between the substrate (cathode) and a Pb-Sb alloy (95-5\%) (anode). The voltage employed was obtained by the Agilent continuous current source, model E3631A.

Table 1. Chemical bath for electrolytic deposition

\begin{tabular}{lc}
\hline Chemical Bath & Concentration \\
\hline $\mathrm{CrO}_{3}$ & $274 \mathrm{~g} / 1$ \\
$\mathrm{H}_{2} \mathrm{SiF}_{6}$ & $0,854 \mathrm{~g} / 1$ \\
\hline
\end{tabular}

Table 2. Parameters for black chromium electrolytic deposition

\begin{tabular}{cccc}
\hline \multirow{2}{*}{$\begin{array}{c}\text { Nomeclature } \\
\text { of the samples }\end{array}$} & \multicolumn{3}{c}{ Electrodeposition parameters } \\
\cline { 2 - 4 } & Voltage (V) & Distance (cm) & Time (min) \\
\hline 1 & 5 & 10 & 10 \\
2 & 5 & 10 & 5 \\
3 & 6 & 10 & 10 \\
4 & 6 & 10 & 5 \\
5 & 5 & 5 & 10 \\
6 & 5 & 5 & 5 \\
7 & 6 & 5 & 10 \\
8 & 6 & 5 & 5 \\
\hline
\end{tabular}

The black chromium films were submitted to UV-VisNIR spectrophotometry using a spectrophotometer by Shimadzu, model UV-2600 operating in the region of 220 $\mathrm{nm}$ to $1400 \mathrm{~nm}$ with measurements of the reflectance using the integrating sphere accessory.

The surface roughness of the films were obtained by the CCI MP non-contact optical profilometer, manufactured by Taylor Hobson, connected to a computerized unit using the Taysurf CCI software (Taylor Hobson) for obtaining and analyzing the data.

The morphological characteristics were observed through a FEI scanning electron microscopy (SEM) with environmental module, model Quanta 450, using the functioning principle of secondary electrons.

After the characterizations mentioned above, the electrodeposited films were covered with an antireflective layer, being therefore, named with the addition of an $+\mathrm{AR}$ suffix in all the produced films, and submitted to the same analysis as before.

\section{Results and Discussion}

\subsection{Spectrophotometry UV-Vis-NIR}

The absorptance levels of the films produced by black chromium electrodeposition are shown in Figure 1.

Figure 1 shows that samples 3, 5, 6, 7 and 8 presented rather similar absorption and behavior (around 90\%) along the spectrum under study. Thus, when analyzing the parameters involved in the production of these five films (Table 2), it is perceptible that the only variable that remained constant in $80 \%$ of the samples was the anode-cathode distance of $5 \mathrm{~cm}$.

This analysis corroborates with the work of Lee ${ }^{10}$ which using the electrodeposition technique with a working distance of $5 \mathrm{~cm}$ between anode and cathode obtained black chromium coatings (prepared in copper substrates coated with nickel) with levels of absorptance between 80 and $90 \%$.

In addition to the influence of the electrodeposition parameters, the fact that the films were electrodeposited on the copper substrate may have boosted absorption levels,

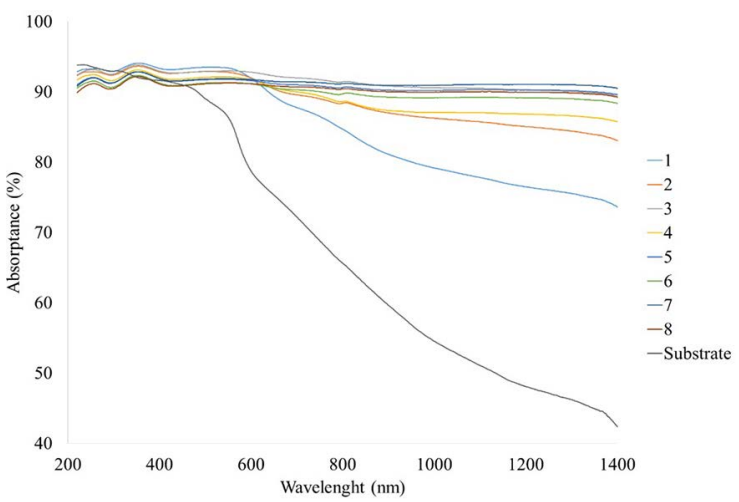

Figure 1. Percentage of absortance of the electrodeposited black chromium films. 
since a better performance of copper compared to other types of substrates is reported in the literature, such as in a study held by Lee et al. ${ }^{6}$, in which black chromium coatings on copper and stainless steel substrates were prepared by electrodeposition in order to investigate their thermal degradation with increasing annealing temperature. They determined that the films produced on the copper substrate showed better performance at absorption levels (91\%) than those deposited on steel (88\%).

In a study held by Farooq and Raja ${ }^{17}$, in which the black chromium coatings were electrodeposited on copper and aluminum substrates, films on the copper substrate also showed the highest levels of absorption. In order to compare the performance of fourteen types of substrates, Smith et al. ${ }^{18}$ realized that while the copper substrate reached absorptance levels of $98 \%$, the steel obtained levels of $95 \%$. This is justified by Vasudevan et al. ${ }^{19}$, who stated that the bath throwing power is improved if the substrate is coated with copper or nickel.

However, the level of absorption of the film can still be improved. By the study of Farooq and Raja ${ }^{17}$ one can understand that this objective can be reached with the use of an AR layer because, when depositing on copper substrates, a film of $\mathrm{Ni}: \mathrm{SiO}_{2}$ obtained an average of $95 \%$ absorption, higher than the electrodeposited film.

Thus, the same black chromium films employed to determine the absorptions expressed in Figure 1 were coated with the AR layer. The result of the absorptances of this set can be analyzed in Figure 2.

For allowing a better analogy between black chromium coatings without and with the AR layer, Figure 3 shows the average absorptance and the respective standard deviations of each film deposited on the copper substrate.

From Figure 3 one can notice that all electrodeposited selective coatings had their absorption levels risen in the order of $1,4 \%$ to $7,8 \%$ after the deposition of the AR layer in addition to having their standard deviations reduced, which guarantees the production of films with more precise absorption levels.

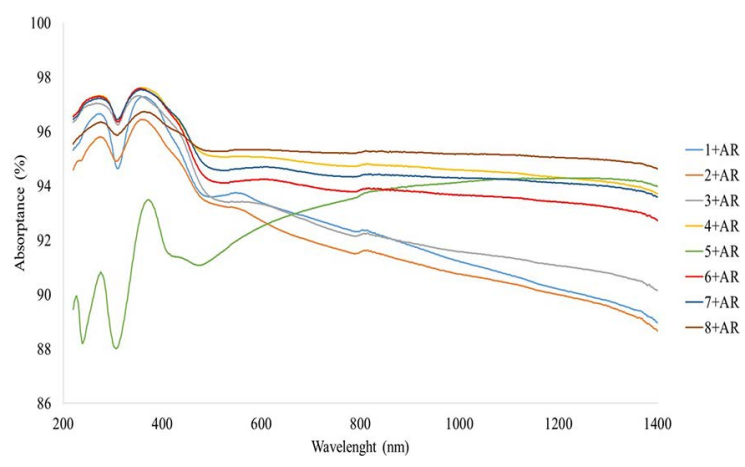

Figure 2. Percentage of absorptance of the black chromium films coated by the AR layer.

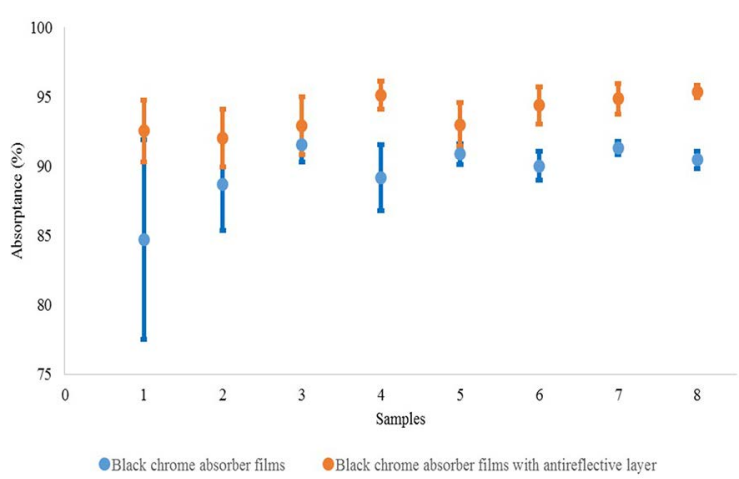

Figure 3. Comparison between the average absorption obtained by the films without and with the AR layer.

It is observed that although the AR layer significantly improved the absorption performance of the electrodeposited films with a working distance of $10 \mathrm{~cm}$, when compared to the films deposited with an anode-cathode distance of $5 \mathrm{~cm}$, those of smaller distance obtained the higher levels of absorption with lower values of standard deviation, evidencing the direct influence of the working distance. In this sense, in the present study, the distance of $5 \mathrm{~cm}$ was shown to be the most adequate to be used, in order to obtain higher absorption levels ${ }^{10}$.

\subsection{Optical Perfilometry}

The obtained films were also submitted surface analysis in the profilometer, without and with the AR layer, as can be seen in Table 3.

In terms of working distance, it can be observed from Table 3 that for a greater distance $(10 \mathrm{~cm})$ the parameter Ra presents smaller values (less roughness), resulting in a lower absorption, besides a high standard deviation. When a smaller working distance $(5 \mathrm{~cm})$ was used, the parameter Ra was enlarged along with the film absorption, besides promoting reduction in the standard deviation of the absorption levels. This evidences the influence of the working distance in obtaining a suitable roughness to the use of the coatings as selective solar absorbers.

This influence can also be corroborated by the microstructural analysis that evidenced the improvement of the quality of films produced with a shorter working distance. This was already expected by the fact that at a lower anode-cathode distance the deposition reaction occurs more easily ${ }^{6}$.

In addition, it was possible to observe that with the insertion of the AR layer, the roughness levels were increased, at all the working distances used, favoring the increase of the absorption. For absorption levels, it is observed that the ideal value for obtaining absorptance over $90 \%$ is with Ra between $0.25 \mu \mathrm{m}$ and $2.5 \mu \mathrm{m}$.

Thus, by Table 3, it can be observed that higher Ra parameter indicates greater film absorption, both without and 
Table 3. Ra parameter of the electrodeposited films over copper substrate, with and without AR layer.

\begin{tabular}{|c|c|c|c|c|c|c|c|}
\hline Samples & $\operatorname{Ra}(\mu \mathrm{m})$ & $\begin{array}{c}\text { Average } \\
\text { absorptance (\%) } \\
(220 \mathrm{~nm}-1400 \mathrm{~nm})\end{array}$ & $\begin{array}{c}\text { Standard } \\
\text { deviation } \\
(\%)\end{array}$ & Samples & $\operatorname{Ra}(\mu \mathrm{m})$ & $\begin{array}{c}\text { Average } \\
\text { absorptance }(\%) \\
(220 \mathrm{~nm}-1400 \mathrm{~nm})\end{array}$ & $\begin{array}{l}\text { Standard } \\
\text { deviation } \\
(\%)\end{array}$ \\
\hline 1 & 0,09 & 84,72 & $\pm 7,18$ & $1+\mathrm{AR}$ & 1,36 & 92,53 & $\pm 2,22$ \\
\hline 2 & 0,13 & 88,68 & $\pm 3,34$ & $2+A R$ & 1,57 & 92,03 & $\pm 2,09$ \\
\hline 3 & 0,27 & 91,52 & $\pm 1,21$ & $3+\mathrm{AR}$ & 1,95 & 92,91 & $\pm 2,10$ \\
\hline 4 & 0,22 & 89,17 & $\pm 2,38$ & $4+\mathrm{AR}$ & 1,76 & 95,10 & $\pm 1,02$ \\
\hline 5 & 0,48 & 90,86 & $\pm 0,76$ & $5+\mathrm{AR}$ & 0,92 & 93,00 & $\pm 1,57$ \\
\hline 6 & 0,28 & 90,03 & $\pm 1,01$ & $6+\mathrm{AR}$ & 2,07 & 94,38 & $\pm 1,35$ \\
\hline 7 & 0,59 & 91,32 & $\pm 0,47$ & $7+A R$ & 2,11 & 94,86 & $\pm 1,09$ \\
\hline 8 & 0,32 & 90,45 & $\pm 0,61$ & $8+\mathrm{AR}$ & 1,80 & 95,37 & $\pm 0,45$ \\
\hline
\end{tabular}

with the AR layer. It is noted that as the Ra parameter were similar and sometimes between the range of wavelengths under study (220 nm - $1400 \mathrm{~nm}$ ) the obtaining of high levels of absorption is possible. This fact can be explained by the optical trap phenomenon.

According to Kennedy ${ }^{3}$, optical traps are capable of stablishing a distinction between the different wavelengths, in a way that the surface appear rugged and absorbent to solar radiation while being mirrored and reflecting to thermal radiation. This may have been the reason why sample 1 did not achieve a good result, while the same film when covered with the AR layer achieved. To better visualize the possibility of optical traps in the surface of these films, Figure 4 illustrates their 3D Profilometry.

According to Cuomo et al. ${ }^{20}$ a surface constituted of an austere forest of aligned needles with diameters in the same dimensions as the visible light wavelengths, with distances between them in the order of many wavelengths, allows an absorption with higher efficiency in function of the multiple reflections occurring as the photons penetrate the labyrinth of needles. Thus, by observing Figure 4, the increasing of the $\mathrm{Rp}, \mathrm{Rv}$ and Ra parameters may have promoted this "labyrinth of needles" construction that favored the absorption.

Through Figure 5 it is noticeable the construction of the optical trap in the surface of the film, being one the of possible causes for the increasing of the absorption.

\subsection{Scanning Electron Microscope (SEM)}

SEM analysis granted the visualization of the films surfaces, allowing the comprehension of their solidification process. The films exposed to Surface Profilometry (Figures 4 and 5) are illustrated in the form of micrographs in Figures 6 and 7 , in order to establish a comparison between the two microstructural characterization techniques.

By the analysis of Figures 6 and 7, one can notice that the electrodeposited films presented cracks. This phenomenon

$$
\begin{aligned}
& \mathrm{Rp}=0,305 \mu \mathrm{m} \\
& \mathrm{Rv}=0,182 \mu \mathrm{m} \\
& \alpha=84,72 \%
\end{aligned}
$$

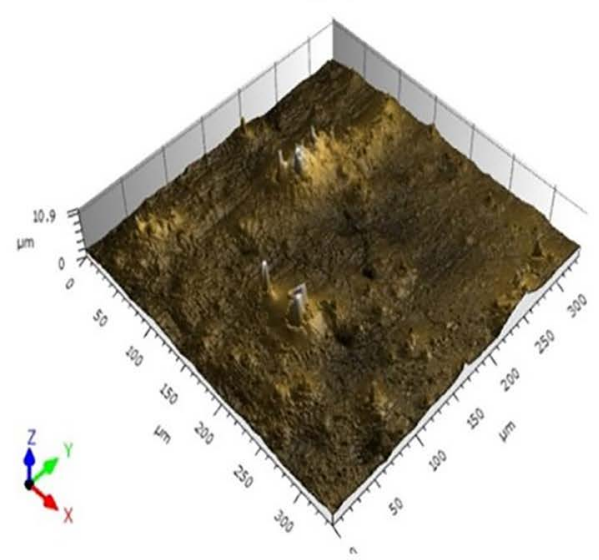

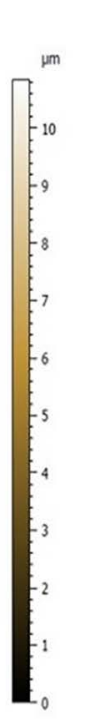

$$
\begin{aligned}
& \mathrm{Rp}=2,92 \mu \mathrm{m} \\
& \mathrm{Rv}=3,66 \mu \mathrm{m} \\
& \alpha=92,53 \%
\end{aligned}
$$

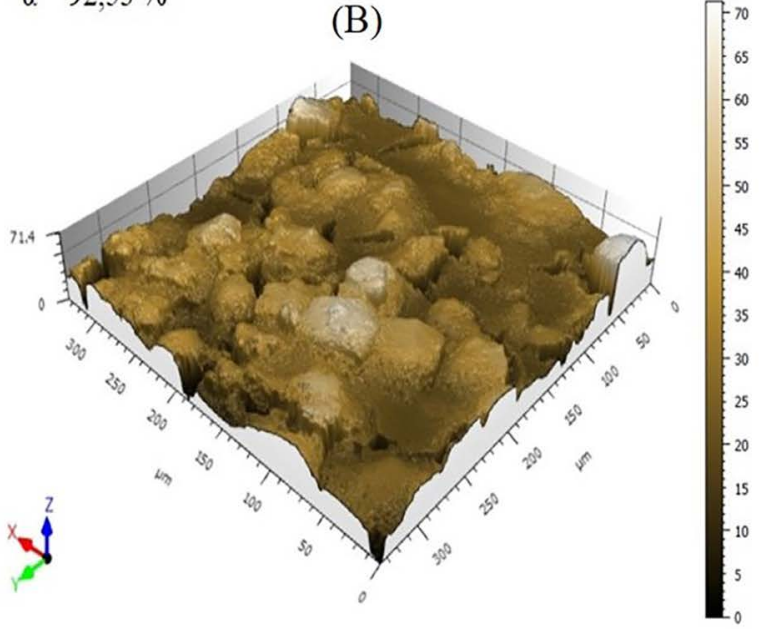

Figure 4. Surface Profilometry of sample 1, without (A) and with (B) the AR layer. 
is common in the black chromium films obtained by the technique of electrodeposition ${ }^{21}$, due to the reduction of hydrogen ions during the process. In addition, it has been found that black chromium has a lamellar morphology leading to a strong level of dispersion, which indicates a higher roughness coefficient of this film and therefore the dark appearance.

The use of the AR layer promoted the modification of the film morphology, as can be seen in Figure 7(b) in which Sample 1 had its Ra parameter elevated from $0.09 \mu \mathrm{m}$ to $1.36 \mu \mathrm{m}$, generating an increase in absorption of $7.8 \%$.

$$
\begin{aligned}
& R p=0,305 \mu \mathrm{m} \\
& R v=0,182 \mu \mathrm{m} \\
& \alpha=84,72 \%
\end{aligned}
$$

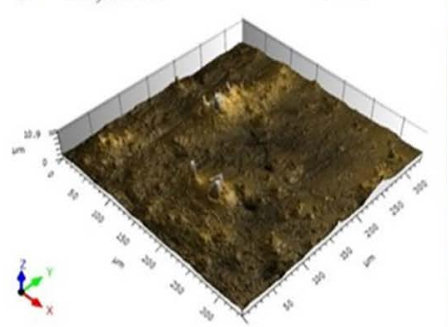

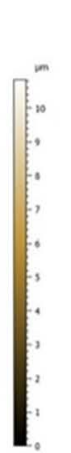

$\mathrm{Rp}=0,473 \mu \mathrm{m}$

$$
\mathrm{Rv}=0,250 \mu \mathrm{m}
$$$$
\alpha=88,68 \%
$$

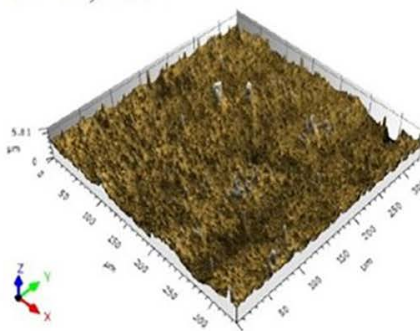

(B)

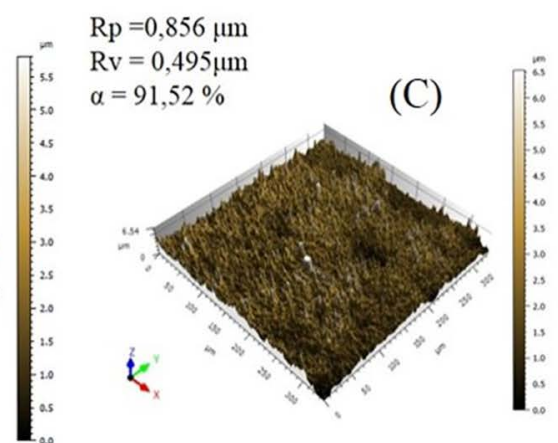

Figure 5. Profilometry of the surface of the films 1(a), 2(b) and 3(c).

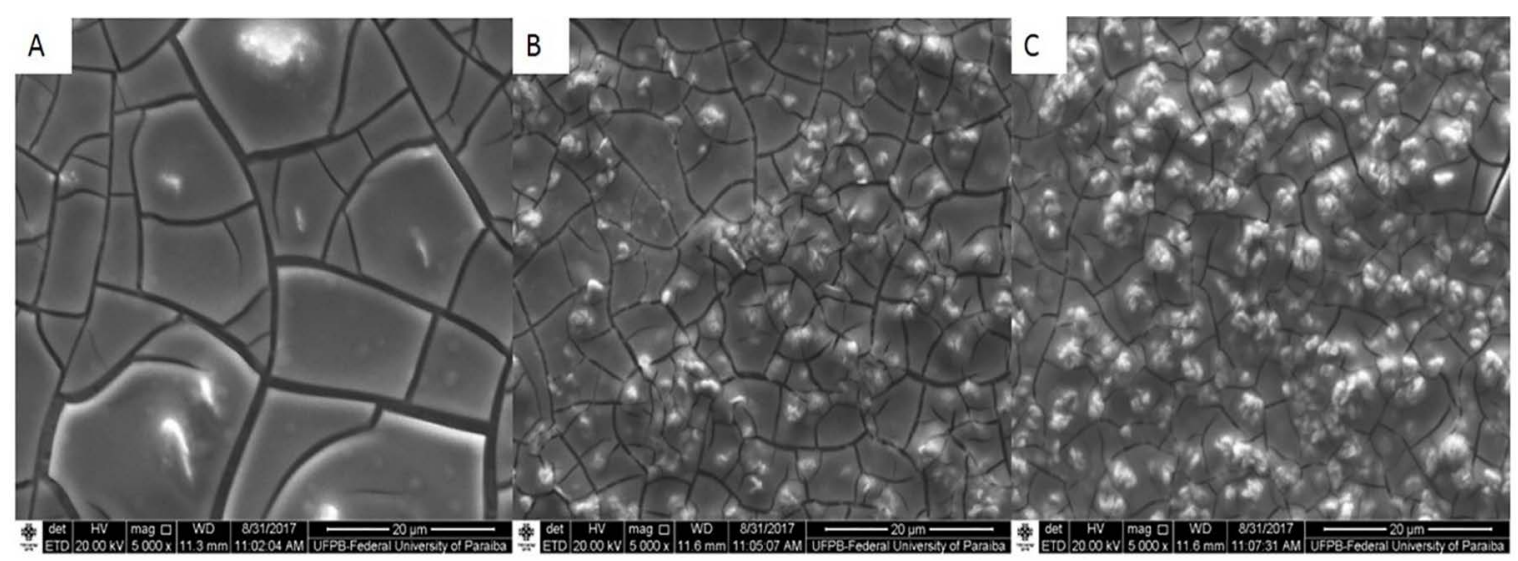

Figure 6. Micrographs of the samples 1 (a), 2 (b) and 3 (c).

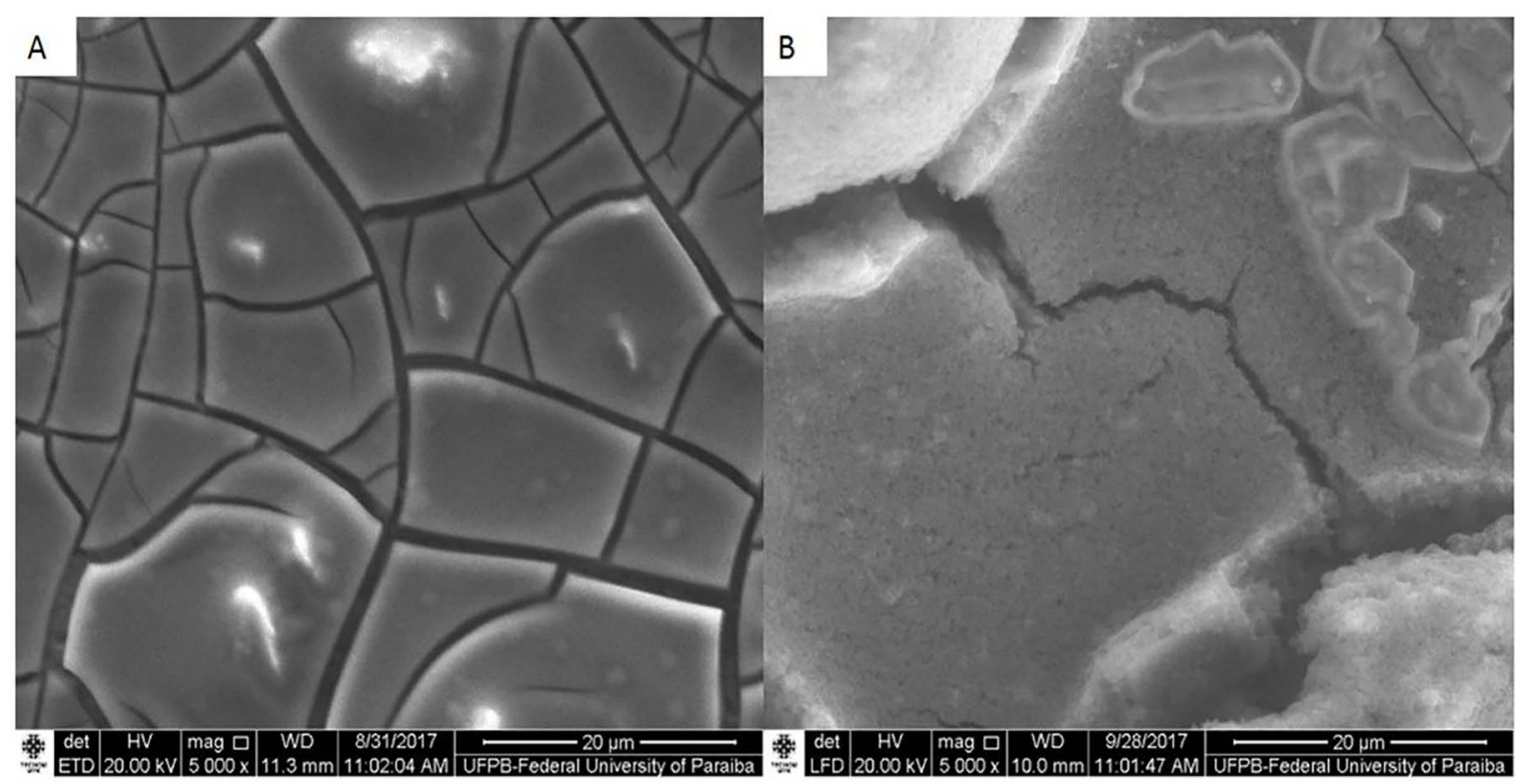

Figure 7. Micrographs of the sample 1 without (a) and with (b) the AR layer. 


\section{Conclusion}

By the present study, in terms of the electrodeposition parameters evaluated, the anode-cathode distance of $5 \mathrm{~cm}$ stimulated the obtaining of higher levels of absorption, as observed in the results of absorption and roughness. In this way, it is possible to conclude that the working distance of 5 $\mathrm{cm}$ made it possible to obtain selective films with appropriate absorption levels (above 90\%), due to the surface roughness (above $25 \mu \mathrm{m}$ ) obtained by the films produced in this work.

The addition of the antireflective layer on the black chromium films favored the increase of the average absorptance in all the adopted set of parameters, besides reducing the standard deviation around the average and increasing the absorption values in the order of up to $7.8 \%$.

As for the microstructure of the obtained films, the increase of the Ra parameter promoted an increase of the absorption, due to the formation of optical traps, which were proved through SEM.

\section{Acknowledgement}

Financial support for the present work was provided by CAPES and CNPq. The authors wish to thank Biomaterials, Rapid Solidification and Synthesis and Characterization of Thin Films Laboratories of the UFPB to assist in the measurements of profilometry, microscopy and UV-Vis-NIR.

\section{References}

1. Chwieduk D. Solar energy utilization. Opto-Electronics Review. 2004;12(1):13-20.

2. Atkinson C, Sansom CL, Almond HJ, Shaw CP. Coatings for concentrating solar systems - A review. Renewable and Sustainable Energy Reviews. 2015;45:113-122.

3. Kennedy CE. Review of Mid- to High-Temperature Solar Selective Absorber Materials - NREL/TP-520-31267. Golden: National Renewable Energy Laboratory; 2002.

4. Trease $\mathrm{CH}$, Hadavinia H, Barrington PE. Solar selective coatings: industrial state-of-the-art. Recent Patents on Materials Science. 2013;6(1):1-19.

5. Cao F, McEnaney K, Chen G, Ren Z. A review of cermet-based spectrally selective solar absorbers. Energy \& Environmental Science. 2014;7(5):1615-1627.

6. Lee KD, Jung WC, Kim JH. Thermal degradation of black chrome coatings. Solar Energy Materials and Solar Cells. 2000;63(2):125-137.
7. Nunes RAX, Costa VC, Sade W, Araújo FR, Silva GM. Selective Surfaces of Black Chromium for Use in Solar Absorbers. Materials Research. 2018;21(1):e20170556.

8. Jeeva PA, Narayanan S, Karthikeyan S. A review on Black coatings for Solar Energy Storaging Systems. International Journal of ChemTech Research. 2016;9(3):589-596.

9. McDonald GE. Spectral reflectance properties of black chrome for use as a solar selective coating. Solar Energy. 1975;17(2):119122.

10. Lee KD. Preparation and characterization of black chrome solar selective coatings. Journal of Korean Physical Society. 2007;51:135.

11. Aguilar M, Barrera E, Palomar-Pardavé M, Huerta L, Muhl S. Characterization of black and white chromium electrodeposition films: surface and optical properties. Journal of Non-Crystalline Solids. 2003;329(1-3):31-38.

12. Kotilainen M, Mizohata K, Honkanen M, Vuoristo P. Influence of microstructure on temperature-induced ageing mechanisms of different solar absorber coatings. Solar Energy Materials and Solar Cells. 2014;120(Pt B):462-472.

13. Lampert CM, Washburn J. Microstructure and optical properties of black chrome before and after exposure to high temperatures. In: Second Annual Conference on Absorber Surfaces for Solar Receivers; 1979 Jan 24-25; Boulder, CO, USA.

14. Raut HK, Ganesh VA, Nair AS, Ramakrishna S. Anti-reflective coatings: A critical, in-depth review. Energy \& Environmental Science. 2011;4(10):3779-3804.

15. de Medeiros IDM, Gomes KC, Silva Neto JF. Viability of Sugarcane Bagasse Ash as Precursor Material to Solar Absorbers Films. Materials Science Forum. 2017;881:427-432.

16. Daryabegy M, Mahmoodpoor AR. Method of Manufacturing Absorbing Layers on Copper for Solar Applications (I). ISECO Science and Technology Vision. 2006;2(1):35-39.

17. Farooq M, Raja IA. Optimisation of metal sputtered and electroplated substrates for solar selective coatings. Renewable Energy. 2008;33(6):1275-1285.

18. Smith GB, Teytz K, Hillery P. The substrate impact on the structure and thermal stability of solar selective black chrome. Solar Energy Materials. 1983;9(1):21-41.

19. Vasudevan N, Grips VKW, Rajagopalan I. The present status of black chromium plating. Surface Technology. 1981;14(2):119132.

20. Cuomo JJ, Ziegler JF, Woodall JM. A new concept for solar energy thermal conversion. Applied Physics Letters. 1975;26(10):557559.

21. Sheu HH, Lu CE, Lee HB, Pu NW, Wu PF, Hsieh SH, et al. Electrodeposition of black chromium-cobalt alloy based on trivalent sulfate electrolyte. Journal of the Taiwan Institute of Chemical Engineers. 2016;59:496-505. 